\title{
Sepsis neonatal en la era de profilaxis antimicrobiana prenatal
}

\author{
José Luis Tapia I., Cristina Reichhard T., M. Isabel Saldías R., Fernando Abarzúa C., \\ M. Eugenia Pérez A., Álvaro González M. y Alessandra Gederlini G.
}

\section{Neonatal sepsis in the era of antenatal antibiotic prophylaxis}

The aim of this study was to compare incidence, bacteriology and associated mortality of neonatal sepsis in a neonatal unit, after (2001-2004) and before (1995-1996) implementation of universal screening for prevention of Group B Streptococcus diseases. Early onset sepsis incidence decreased from 2.5 to 1 per 1000 live births $(p=0.03)$, with a decrease in the proportion of Group B Streptococcus cases from a $54 \%$ to $11 \%$ $(\mathrm{p}<0.01)$. In late onset sepsis, coagulase-negative Staphylococci continued to be the predominant pathogen (49\% of cases), and Candida albicans emerged as the second etiologic agent in the postscreening period. Sepsis associated mortality was low in both periods $(2.2 \%)$.

Key words: Neonatal sepsis, Newborn infants, Bacteriology, Streptococcus agalactiae (group B).

Palabras claves: Sepsis neonatal, Recién nacidos, Bacteriología, Streptococcus agalactiae (grupo B).
Pontificia Universidad Católica de Chile, Santiago, Chile Hospital Clínico Departamento de Pediatría, Unidad de Neonatología (JLTI, MISR, MEPA, AGM)

Departamento Ginecología y Obstetricia (FAC)

Departamento Salud Pública (AGG) Escuela de Medicina Estudiante de Medicina (CRT)

Recibido: 27 enero 2006 Aceptado: 21 septiembre 2006

Correspondencia a: José Luis Tapia Illanes jlta@med.puc.cl

\section{Introducción}

$\mathrm{L}$ a sepsis de origen bacteriano constituye una de las principales causas de morbimortalidad en el período neonatal. Es causada por microorganismos que han variado a través del tiempo debido a estrategias globales de prevención por una parte, y por otra, a las características propias de los recién nacidos (RN) hospitalizados, procedimientos y tratamientos que ellos reciben ${ }^{1-3}$.

Los mecanismos de inmunidad de los recién nacidos son relativamente deficientes en comparación con lactantes y niños mayores, siendo esto de especial relevancia en RN prematuros y de bajo peso de nacimiento en los cuales, la inmadurez del sistema inmune se acompaña de otros factores de riesgo y de procedimientos invasores que aumentan la vulnerabilidad a infecciones ${ }^{2,3}$.

Según el momento de aparición se clasifican en sepsis precoz, la cual es, generalmente, de origen connatal y sepsis tardía (posterior a 72 horas de vida para la mayoría de los autores), las que se consideran como fundamentalmente intrahospitalarias ${ }^{2,5}$.

La incidencia y letalidad de la septicemia neonatal es variable, reportándose una incidencia entre 2 y $4 /$ 1.000 nacidos vivos en países desarrollados, hasta 10/ 1.000 nacidos vivos en otras series. La letalidad reportada oscila entre menos de 10 y $70 \%{ }^{6-9}$.
La bacteriología de las infecciones en RN ha variado en forma considerable en las últimas décadas, tanto en infecciones connatales (en el contexto de estrategias de prevención implementadas), como en infecciones nosocomiales, en relación a la bacteriología de cada unidad neonatal, resistencia antimicrobiana, procedimientos invasores, etc ${ }^{10-15}$.

Desde la década de 1970 Streptococcus agalactiae (grupo B) es el patógeno más relevante en infecciones connatales, asociándose a una mortalidad entre 5 y $20 \%$ y a secuelas en $30 \%$ de los sobrevivientes. La población de mayor riesgo para presentar sepsis por $S$. agalactiae es la de RN de pretérmino, hijos de madres con antecedentes de sepsis neonatal en embarazos anteriores, con infección urinaria por $S$. agalactiae en el embarazo actual, rotura prematura de membranas de más de 18 horas o con fiebre intraparto. Sin embargo, hasta $50 \%$ de las sepsis connatales por S. agalactiae se presentan en RN de término hijos de madres colonizadas pero sin dichos factores de riesgo ${ }^{16-18}$.

La prevalencia reportada de colonización asintomática por S. agalactiae en el tercer trimestre del embarazo varía entre 2 y $34 \%$. En Chile, la portación de $S$. agalactiae se acerca a $20 \%{ }^{7,19}$. Uno a $2 \%$ de los RN de madres colonizadas desarrollarán sepsis ${ }^{20,21}$.

En 1996, el CDC de Atlanta, E.U.A., en conjunto con el Colegio Americano de Obstetricia y Ginecología (ACOG) y la Academia Americana de Pediatría (AAP) 
publicaron las guías para la prevención de sepsis por S. agalactiae. En ese momento propusieron dos estrategias de prevención igualmente aceptables: una basada sólo en factores de riesgo, y la otra agregaba el cultivo universal a las 35-37 semanas de gestación. Los resultados de la implementación de estas estrategias concluyeron que la utilización de factores de riesgo previene alrededor de $68 \%$ de la sepsis por $S$. agalactiae y que la incorporación de cultivo puede tener una eficacia de $88 \%$, disminuyendo la incidencia de 1,7 a 0,4/1.000 nacidos vivos entre 1993 y 199922-24. Por ello, el CDC modificó en 2002 su normativa y propuso aplicar sólo el protocolo basado en el cultivo universal a las madres gestantes ${ }^{25,26}$.

Se desconoce si la estrategia de prevención prenatal puede tener algún efecto en la incidencia de sepsis neonatal tardía. En una publicación reciente se sugiere que podría aumentar la gravedad de las infecciones tardías $^{27}$. Por otra parte, reducir la incidencia de sepsis intrahospitalaria es un desafío prioritario, en especial en el RN de muy bajo peso. Aproximadamente $20 \%$ de esta población presenta una sepsis tardía ${ }^{28}$. Además del riesgo de mortalidad, ésta se asocia a un mayor riesgo de morbilidad aguda como ductus arterioso persistente, y tardía como displasia broncopulmonar ${ }^{29}$. La relevancia de este problema exige una vigilancia clínica y epidemiológica estricta y permanente en cada Unidad Neonatal.

El objetivo de este estudio fue determinar la incidencia, mortalidad y etiología de las sepsis neonatales en el período 2001-2004 en nuestra unidad de neonatología (protocolo con cultivo universal, el cual se implementó gradualmente entre los años 1997 y 2000), comparando los resultados con el período 1995 -1996 (sin estrategias preventivas).

\section{Pacientes y Métodos}

Se incluyó a todos los $\mathrm{RN}$ hospitalizados en la Unidad de Cuidados Intensivos Neonatal del Hospital Clínico de la Pontificia Universidad Católica de Chile que tuvieron hemocultivo positivo durante el período 2001 a 2004.

Durante ese período se siguieron las recomendaciones de la CDC:

- Cultivo vaginal y rectal selectivo para pesquisar $S$. agalactiae a las 35-37 semanas a toda mujer embarazada, excepto que la paciente presentara bacteriuria por $S$. agalactiae en el embarazo actual; o que existiera el antecedente de enfermedad invasora por S. agalactiae en un hijo previo.

Se indicó profilaxis antimicrobiana intraparto si existía:
- Bacteriuria por S. agalactiae en el embarazo actual.

- Antecedente de enfermedad invasora por $S$. agalactiae en un hijo previo.

- Resultado positivo del tamizaje en el embarazo actual (excepto si el parto se resolviera por cesárea electiva y en presencia de membranas íntegras).

Si el resultado del tamizaje era desconocido, se administró profilaxis si:

- El trabajo de parto se desencadenó antes de las 37 semanas.

- Hubo rotura de membranas por un período mayor o igual a 18 horas.

- Hubo fiebre mayor o igual a $38^{\circ} \mathrm{C}$ intraparto.

Se consideró sepsis precoz si el hemocultivo fue positivo en las primeras 72 horas de vida y sepsis tardía si fue positivo después de las 72 horas y hasta el alta.

Se excluyeron pacientes trasladados desde otras unidades, de manera que todos los casos de sepsis precoz correspondieron a $\mathrm{RN}$ provenientes de la maternidad institucional.

Se consideró mortalidad asociada a sepsis si ella se produjo dentro de los primeros siete días post cultivo positivo o cuando clínicamente se consideró que ésta fue la causa desencadenante de muerte ${ }^{30}$.

El resultado de hemocultivos se registró en forma rutinaria. Las características de la población estudiada, así como la relación con procedimientos invasores, se extrajeron en forma retrospectiva de las fichas clínicas.

Se compararon los resultados poblacionales y la bacteriología con el período 1995-1996. Se analizaron las características poblacionales, edad de presentación y relación con procedimientos invasores.

Para la comparación de variables continuas se utilizó test de Kruskal- Wallis y para variables discretas test exacto de Fisher. Se consideró significativo un valor $\mathrm{p}<0,05$.

\section{Resultados}

En el período estudiado hubo 9.002 partos, habiéndose efectuado cultivo según el protocolo a $90 \%$ de las madres y diagnosticándose sepsis neonatal en 46 $\mathrm{RN}$. Las características poblacionales de los $\mathrm{RN}$ que presentaron sepsis neonatal en los dos períodos son comparables. El promedio de peso de nacimiento (PN) en el período 2001-2004 fue de $1.760 \pm 1.071$ grs y PN $1.900 \pm 1.098$ en el período anterior. El promedio de edad gestacional de los RN estudiados fue de 31,2 \pm 5,7 semanas en el último período y de $32,5 \pm 5$ semanas en el período 1995-1996. La distribución de RN 
por sexos también fue comparable en los dos grupos. La letalidad por sepsis fue de $2,2 \%$ en ambos períodos.

La incidencia y etiología de sepsis precoz se muestra en la Tabla 1. Los hemocultivos tuvieron un rendimiento de 8,9\% en el período 2001 -2004 en comparación con 7,5\% en el período $1995-1996(\mathrm{p}=0,4)$. Al comparar la incidencia de sepsis precoz se observó una disminución de ésta en el período 2001-2004 $(\mathrm{p}=0,03)$, junto a una importante reducción de casos de sepsis causadas por $S$. agalactiae $(\mathrm{p}=0,01)$. De hecho, sólo se presentó un caso de infección connatal por $S$. agalactiae en el período con profilaxis antibiótica prenatal, un recién nacido de término, en el cual el tamizaje fue negativo por lo que no recibió profilaxis.

El número de casos y la etiología de la sepsis tardía aparecen en la Tabla 2. No es posible comparar resultados de incidencia por sepsis tardía en nuestra unidad dado que el número de pacientes ingresados por traslado ha aumentado mucho en el tiempo y están incluidos en el análisis. Staphylococcus coagulasa negativa (SCN) se mantuvo como microorganismo predominante en la sepsis tardía, destacando la aparición de sepsis por Candida albicans en el período más reciente.

La edad de aparición de la sepsis tardía fue a los 19 días (mediana) con rango entre 4 y 124 días. El número de episodios según edad de aparición se muestra en la Figura 1. El 45\% de los casos de sepsis tardía se asoció a procedimientos invasores. Estos se presentaron a los 11 días (mediana) de cateterismo, con un rango entre 2 y 45 días; y a los 11 días (mediana) de ventilación mecánica, con un rango entre 2 y 31 días.

\section{Discusión}

En los últimos diez años, la mayor utilización de protocolos de prevención de sepsis neonatal, con administración de antimicrobianos intraparto, ha producido reducciones significativas de la enfermedad precoz, con tasas estables del cuadro tardío. El mayor impacto en dicha reducción ha sido reportado con protocolos basados en un tamizaje universal con cultivo al término del embarazo, por sobre los protocolos más simples, que se basan en la identificación de factores de riesgo ${ }^{31}$.

Nuestro centro incorporó entre los años 1999 y 2000 el protocolo con cultivo selectivo (caldo Todd Hewitt) vaginal y anal a todas las mujeres embarazadas, reportando una prevalencia de $20 \%$ de mujeres gestantes colonizadas al final del embarazo ${ }^{7}$. El presente estudio analiza la tasa de sepsis neonatal (precoz y tardía) en el período de protocolo con cultivo

\begin{tabular}{|c|c|c|}
\hline & 1995-1996 & 2001-2004 \\
\hline $\mathrm{n} / \mathrm{n}^{\circ}$ partos & $13 / 5.170$ & $9 / 9.002$ \\
\hline Incidencia* (x/1.000 RNV) & 2,5 & 1,0 \\
\hline \multirow[t]{3}{*}{ Etiología } & Streptococcus agalactiae 54\%** & Streptococcus agalactiae $11 \%$ \\
\hline & Escherichia coli $\quad 0 \%$ & Escherichia coli $\quad 22 \%$ \\
\hline & Listeria monocytogenes & Listeria monocytogenes 11\% \\
\hline
\end{tabular}

Tabla 2. Número de casos y etiología de sepsis tardía en dos períodos. Hospital Clínico Universidad Católica de Chile

\begin{tabular}{|c|c|c|c|}
\hline & 1995-1996 & & 2001-2004 \\
\hline $\mathrm{N}^{\circ}$ de casos & 32 & & 37 \\
\hline \multirow[t]{3}{*}{ Etiología } & Staphylococcus coagulasa (-) & $59 \%$ & Staphylococcus coagulasa (-) $49 \%$ \\
\hline & Staphylococcus aureus & $34 \%$ & Candida albicans \\
\hline & & & Staphylococcus aureus \\
\hline
\end{tabular}

Nota: no incluye número de partos ni incidencia de sepsis por existir un número importante de traslados de otros centros en la serie

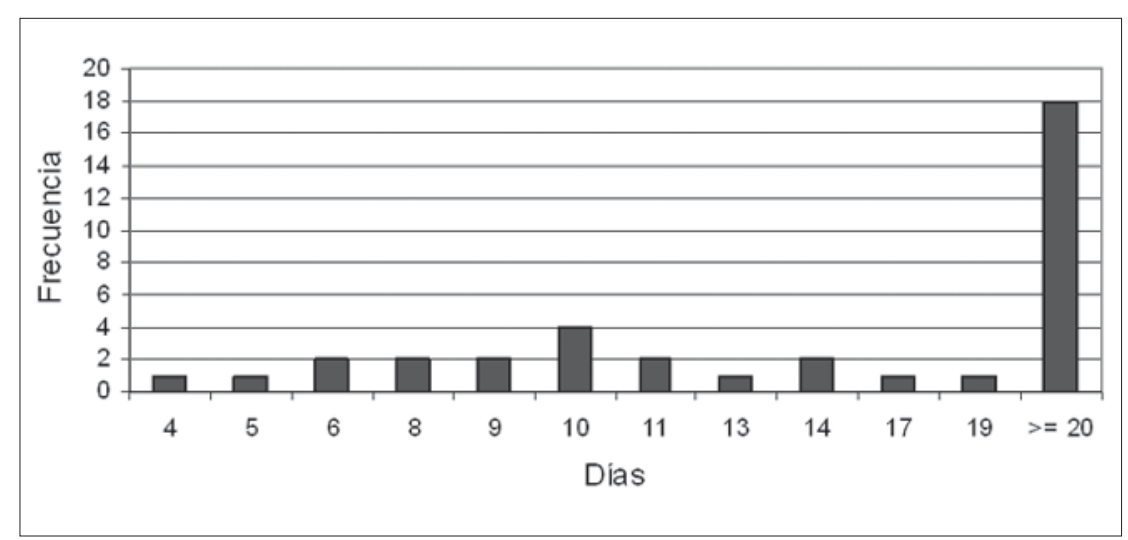

Figura 1. Edad de presentación de la sepsis tardía: número de episodios de sepsis tardía según edad de aparición.

universal y la compara con la tasa de períodos anteriores, en la que no se realizaba prevención.

Destacamos la disminución significativa de casos de sepsis precoz por $S$. agalactiae a 0,1/1.000 RN vivos y además una tendencia a la disminución global de las sepsis precoces, con la tasa más baja de los últimos 18 años (1/1.000 RN vivos). Desde el inicio del protocolo con tamizaje universal en nuestro servicio, 
sólo se ha presentado un caso de infección connatal por $S$. agalactiae, un RN de término en el cual el tamizaje fue negativo por lo que no recibió profilaxis (probable adquisición post cultivo).

En períodos anteriores al año 2000, S. agalactiae fue la especie microbiana preponderante con una incidencia mayor a $50 \%$ de los casos. En el período más reciente existe una etiología más variada para la sepsis precoz. Se observó una mayor frecuencia de Escherichia coli, seguida de S. agalactiae y Listeria monocytogenes que se ha mantenido con incidencia similar en los tres períodos. Los cambios en la incidencia y etiología de la sepsis precoz observados en nuestra unidad concuerdan con los datos reportados en múltiples publicaciones extranjeras desde el inicio de la profilaxis antimicrobiana como prevención de sepsis por $S$. agalactiae, confirmando su efectivi$\operatorname{dad}^{3,22,23,30,32,33}$. De acuerdo con las cifras del Colegio Americano de Obstetricia y Ginecología, la incidencia de sepsis por $S$. agalactiae en E.U.A. disminuyó a 0,23/1.000 RN vivos ${ }^{26}$. La presentación de una sepsis por $S$. agalactiae en nuestro centro, a pesar de la estrategia preventiva, sólo confirma que ésta no es $100 \%$ protectora, pudiendo la madre adquirir el microorganismo con posterioridad a la semana del tamizaje como probablemente ocurrió en nuestro caso.

La prevención de sepsis por $S$. agalactiae con cultivo prenatal ha sido implementada en Chile, en nuestra unidad y en otros centros privados. Esta estrategia no ha sido incorporada aún en los programas del Ministerio de Salud y no se han publicado estudios de costo efectividad de estas medidas en nuestro país ni en otros países sudamericanos. Publicaciones de otras latitudes calculan menores costos con la implementación del cultivo prenatal que con el uso de estrategias basadas en factores de riesgo, disminuyendo el número de $\mathrm{RN}$ con sepsis precoz por $S$. agalactiae y muerte ${ }^{34-38}$. Estos estudios son necesarios en nuestro país para determinar las estrategias de prevención más adecuadas para la realidad chilena.

En las sepsis tardías se mantiene como microorganismo preponderante a SCN desde fines de la década de los ochenta, destacando la aparición de casos de sepsis por C. albicans en el período 2001-2004. Un porcentaje importante de las sepsis tardías se asocia a procedimientos invasores, con diagnóstico de sepsis a los 11 días (mediana) de cateterismo y ventilación mecánica. La persistencia de SCN como patógeno principal en la sepsis tardía, así como la aparición de sepsis por C. albicans y otros agentes oportunistas, ha sido descrito en otras series ${ }^{8,39}$. Esto no se explicaría por cambios en la virulencia de estos agentes, sino por las características de los RN. La sobrevida actual de RN de muy bajo peso de nacimiento y prematurez extrema nos enfrenta a una población con un sistema inmune extremadamente inmaduro, con morbilidad importante que suele requerir terapias invasoras prolongadas como líneas vasculares con nutrición parenteral y/o soporte ventilatorio. Esto, sumado a terapias antimicrobianas agresivas, los hace susceptibles a infecciones nosocomiales por microorganismos oportunistas.

En relación con la edad de presentación de las sepsis tardías, puede ser de interés conocer esta información, ya que los factores de riesgo serían distintos si ésta se presenta antes o después de los diez días de edad $^{13}$. Además, se puede especular que mientras más tardíamente se presenta, reflejaría mejores medidas preventivas de infección en el cuidado de enfermería. En un estudio recientemente publicado con datos de una unidad neonatal de Nueva York, la mediana fue de 18 días, muy similar a la nuestra ${ }^{40}$.

El porcentaje de hemocultivos positivos encontrado en este estudio fue inferior a $10 \%$. Este resultado es concordante con $9,2 \%$ reportado recientemente ${ }^{3}$, aunque se han descrito cifras tan bajas como $2,7 \%$ en hemocultivos realizados para pesquisar sepsis pre$\mathrm{Coz}^{40}$. Estos bajos porcentajes pueden explicarse, en parte, por tratamientos antimicrobianos administrados a la madre; además los signos iniciales de sepsis en un RN pueden ser bastante inespecíficos, lo cual determina que en la práctica se plantee con gran frecuencia una sepsis como diagnóstico posible en la edad neonatal.

La mortalidad asociada a sepsis ha disminuido en nuestra unidad a través del tiempo ${ }^{1}$ y las actuales cifras son comparables a lo reportado en series de países desarrollados ${ }^{3,6,30}$. Esto puede ser explicado por la disminución de la incidencia y mortalidad por sepsis precoz atribuibles a la exitosa estrategia de prevención de sepsis por $S$. agalactiae y a que los microorganismos comensales de mayor prevalencia actual en sepsis tardías tienen un bajo riesgo de mortalidad. En consecuencia, la implementación de la estrategia de prevención antimicrobiana prenatal no aumentó la gravedad de las sepsis neonatal tardías. Por último, la disminución de la mortalidad por sepsis neonatal también puede estar reflejando la caída en la mortalidad neonatal global, atribuible a los avances en la medicina perinatal.

El presente estudio tiene limitaciones, en especial por corresponder a una casuística baja en número. Además, las cifras de mortalidad asociada a sepsis neonatal en nuestro centro eran bajas previamente a la implementación de la profilaxis antimicrobiana prenatal, como para poder demostrar una caída en ésta. No obstante, nuestros resultados de disminución de la sepsis precoz y de casos de $S$. agalactiae con esta estrategia son consistentes con los de la literatura 
científica extranjera y ratifican la importancia de mantener en cada unidad un seguimiento etiológico de las sepsis neonatales y de los resultados de medidas preventivas.

\section{Resumen}

El objetivo de este estudio fue determinar la incidencia, bacteriología y mortalidad de la sepsis neonatal entre los años 2001-2004 en una unidad neonatal, período en que se utilizó screening universal para prevención de Streptococcus agalactiae (grupo B), en comparación a un período anterior sin estrategias preventivas (1995-96). La incidencia de sepsis precoz disminuyó desde 2,5 a 1/1.000 nacidos vivos $(\mathrm{p}=0,03)$, observándose una disminución de casos de $S$. agalactiae de 54 a $11 \%(\mathrm{p}<0,01)$. En sepsis tardías, Staphylococcus coagulasa negativa permanece como el patógeno preponderante (49\% de casos), apareciendo Candida albicans como segundo microorganismo causal más frecuente en el último período. La mortalidad asociada a sepsis ha permanecido baja en ambos períodos $(2,2 \%)$. Los resultados de este estudio son concordantes con publicaciones extranjeras.

\section{Referencias}

1.- Concha M, Azócar M, Letelier C, Chicharro A, Saldías M, Tapia J. Septicemia neonatal: incidencia, letalidad y características bacteriológicas. Comparación de dos períodos. Rev Chil Pediatr 1998; 69: 14851.

2.- Tapia J, Prado P. Infecciones Bacterianas. Tapia J L, Ventura-Juncá P. Manual de Neonatología, II Edición, Santiago, Editorial Mediterráneo, año 2000. Págs 227-240.

3.- Gerdes J S. Diagnosis and management of bacterial infections in the neonate. Pediatr Clin North Am 2004; 51: 939-59.

4.- Stoll B. The global impact of neonatal infection. Clin Perinatol 1997; 24: 1-15.

5.- Craft A, Finer N. Nosocomial coagulase negative staphylococcal (CoNS) catheterrelated sepsis in preterm infants: definition, diagnosis, prophylaxis, and prevention. $\mathrm{J}$ Perinatol 2001; 21: 186-92.

6.- Lukacs S, Schoendorf K, Schuchat A. Trends in sepsis-related neonatal mortality in the United States, 1985-1998. Pediatr Infect Dis J 2004; 23: 599-603.

7.- Abarzúa F, Guzmán A, Belmar C, Becker J, García P, Rioseco A, et al. Prevalencia de colonización por Streptococcus agalactiae (grupo B) en el tercer trimestre del embarazo. Evaluación del cultivo selectivo. Experiencia en 2192 pacientes. Rev Chil Obstet Ginecol 2002; 67: 89-93.

8.- Gladstone I, Ehrenkranz R, Edberg S, Baltimore RS. A ten-year review of neonatal sepsis and comparison with the previous fifty-year experience. Pediatr Infect Dis J 1990; 9: 819-25.

9.- Guzmán A, Abarzúa F, Belmar C, García P. Resultados de la aplicación del protocolo basado en screening para la búsqueda de Streptococcus agalactiae en el tercer trimestre del embarazo. Posible impacto sobre la sepsis neonatal precoz por este agente. Rev Chil Infectol 2001; 18: 187-92. 10.- Zaoutis T, Greves M, Lautenbach E,
Bilker W B, Coffin S E. Risk factors for disseminated candidiasis in children with candidemia. Pediatr Infect Dis J 2004; 23 : 635-41.

11.- Nalini S, Sprague B, Patel K, Pollack. Risk factors for nosocomial infection in critically ill children: A prospective cohort study. Crit Care Med 1996; 24: 875-8.

12.- Rubin L, Sánchez P, Siegel J, Levine G, Saiman L, Jarvis W. Evaluation and treatment of neonates with suspected lateonset sepsis: A survey of neonatologists' practices. Pediatrics 2002; 110: e42

13.- Carrieri M, Stolfi I, Moro M. Intercenter variability and time of onset: two crucial issues in the analysis of risk factors for nosocomial sepsis. Pediatr Infect Dis J 2003; 22: 599-609.

14.- Camara D. Minimizing risks associated with peripherally inserted central catheters in the NICU. MCN Am J Matern Child Nurs. 2001; 26: 17-21; quiz 22. Review.

15.- Belmar C, Abarzúa F, Becker J, Guzmán A, García P, Oyarzún E. Estudio de sensibilidad antimicrobiana de 183 cepas de Streptococcus agalactiae aisladas en región vagino-perineal de embarazadas en el tercer trimestre. Rev Chil Obstet Ginecol 2002; 67: 106-9.

16.- Benitz W, Gould J, Druzin M. Risk factors for early-onset group B streptococcal sepsis: estimation of odds ratios by critical literature review. Pediatrics 1999; 103: e77

17.- Rouse D J, Goldenberg R L, Cliver S P, Cutter G R, Mennemeyer S T, Fargason C A Jr. Strategies for prevention of early onset neonatal group B streptococcal sepsis: A decision analysis. Obstet Gynecol 1994; 83: 483-93.

18.- Gibbs R, Schrag S, Schuchat A. Perinatal infections due to group B streptococci. Obstet Gynecol 2004; 104 (5 Pt 1): 106276.

19.- Abarzúa F, Zajer C, Guzmán A, Belmar C, Becker J, Rioseco A, et al. Determinación de la portacion de Streptococcus agalactiae (grupo B) en embarazadas durante el tercer trimestre mediante inmunoensayo. Rev Chil Obstet Ginecol 2002; 67: 293-5.

20.- McKenna D S, Iams J D. Group B streptococcal infections. Semin Perinatol 1998; 22: 267-76.

21.- Centers for Disease Control and Prevention. Prevention of perinatal group B streptococcal disease: a public health perspective. MMWR Morbid Mortal Wkly Rep 1996; 45 (RR7): 1-24.

22.- Schrag S, Zywicki S, Farley M M, Reingold A L, Harrison L, Lefkowitz L, et al. Group B streptococcal disease in the era of intrapartum antibiotic prophylaxis. $\mathrm{N}$ Engl J Med 2000; 342: 15-20.

23.- Platt J S, O'Brien W F. Group B streptococcus: prevention of early-onset neonatal sepsis. Obstet Gynecol Surv 2003; 58: 191-6.

24.- Committee on Infectious Diseases and Committee on Fetus and Newborn. Revised guidelines for prevention of early-onset group B streptococcal (GBS) infection. Pediatrics 1997; 99: 489-96.

25.- Centers for Disease Control and Prevention. Prevention of perinatal group B streptococcal disease. MMWR Morbid Mortal Wkly Rep 2002; 51 (RR 11): 1.

26.- American College of Obstetricians and Gynecologists. ACOG Committee Opinion: number 279, December 2002. Prevention of early-onset group B streptococcal disease in newborns. Obstet Gynecol 2002; 100: 1405 12.

27.- Glasgow T S, Young P C, Wallin J, Kwok C, Stoddard G, Firth $\mathrm{S}$ et al. Association of intrapartum antibiotic exposure and late onset serious bacterial infections in infants. Pediatrics 2005; 116: 696-702.

28.- Stoll B, Hansen N, Fanaroff AA, Wright L L, Carlo W, Ehrenkranz R A, et al. Late onset sepsis in very low birth weight neonates: the experience of the NICHD neonatal research network. Pediatrics 2002; 110: 285-91. 
29.- González A, Sosenko Y, Chandar J, Hummler H, Claure N, Bancalari E. Influence of infection on patent ductus arteriosus and chronic lung disease in premature infants weighing $1000 \mathrm{~g}$ or less. J Pediatr 1996; 128: 470-8.

30.- Bizzarro M, Raskind C, Baltimore R, Gallagher P. Seventy-five years of neonatal sepsis at Yale: 1928-2003. Pediatrics 2005; 116: 595-602.

31.- Puopolo K M, Madoff L C, Eichenwald E C. Early-onset group B streptococcal disease in the era of maternal screening. Pediatrics 2005; 115: 1240-6.

32.- Daley A, Isaacs D and the Australasian Study Group for Neonatal Infections. Ten-year study on the effect of intrapartum antibiotic prophylaxis on early onset group B Steptococcal and Escherichia coli neonatal sepsis in Australia. Pediatr Infect Dis J 2004; 23: 630-4.

33.- López Sastre J, Fernández C, Coto C,
Ramos A. Trends in the epidemiology of neonatal sepsis of vertical transmission in the era of group B streptococcal prevention. Acta Paediatr 2005; 94: 451-7.

34.- Larcher J S, Capellino F, De Giusto R, Travella C, Balangione F G, Kreiker G, et al. Group B streptococcus colonization during pregnancy and prevention of early onset of disease. Medicina (B Aires) 2005; 65: 201-6.

35.- Akker-van Marle M E, Rijnders M E, Dommelen P, Fekkes M, Wouwe J P, Amelink-Verburg M P, et al. Costeffectiveness of different treatment strategies with intrapartum antibiotic prophylaxis to prevent early-onset group B streptococcal disease. Br J Obstetr Gynecol 2005; 112: 820-6.

36.- Stan C M, Boulvain M, Bovier P A, Auckenthaler R, Berner M, Irion $\mathrm{O}$. Choosing a strategy to prevent neonatal early-onset group B streptococcal sepsis: economic evaluation. Br J Obstetr Gynecol
2001; 108: 840-7.

37.- Mohle-Boetani J C, Lieu T A, Ray G T, Escobar G. Preventing neonatal group B streptococcal disease: cost-effectiveness in a health maintenance organization and the impact of delayed hospital discharge for newborns who received intrapartum antibiotics. Pediatrics 1999; 103: 703-10.

38.- Haberland C A, Benitz W E, Sanders G D, Pietzsch J B, Yamada S, Nguyen L, et al. Perinatal screening for group B streptococci: cost-benefit analysis of rapid polymerase chain reaction. Pediatrics 2002; 110: 471-80.

39.- Chapman R, Faix R. Persistent bacteremia and outcome in late onset infection among infants in a neonatal intensive care unit. Pediatr Infect Dis J 2003; 22: 17-21.

40.- Sarkar S, Bhagat I, Decristofaro J D, Wiswell T E, Spitzer A R. A study of the role of multiple site blood cultures in the evaluation of neonatal sepsis. J Perinatol 2006; 26: 18-22. 